\title{
New Fixed Point Theorem on Triple Controlled Metric Type Spaces with Applications to Volterra-Fredholm Integro-Dynamic Equations
}

\author{
Kalpana Gopalan ${ }^{1, *(\mathbb{D})}$, Sumaiya Tasneem Zubair ${ }^{1}(\mathbb{D})$, Thabet Abdeljawad ${ }^{2,3, *(\mathbb{D})}$ and Nabil Mlaiki $^{2}(\mathbb{D})$ \\ 1 Department of Mathematics, Sri Sivasubramaniya Nadar College of Engineering, Kalavakkam, \\ Chennai 603 110, India; sumaiyatasneemz@ssn.edu.in \\ 2 Department of Mathematics and Sciences, Prince Sultan University, P.O. Box 66833, \\ Riyadh 11586, Saudi Arabia; nmlaiki@psu.edu.sa \\ 3 Department of Medical Research, China Medical University, Taichung 40402, Taiwan \\ * Correspondence: kalpanag@ssn.edu.in (K.G.); tabdeljawad@psu.edu.sa (T.A.)
}

Citation: Gopalan, K.; Zubair, S.T.; Abdeljawad, T.; Mlaiki, N. New Fixed Point Theorem on Triple Controlled Metric Type Spaces with Applications to Volterra-Fredholm Integro-Dynamic Equations. Axioms 2022, 11, 19. https://doi.org/ 10.3390/axioms11010019

Academic Editor: Chris Goodrich

Received: 19 November 2021 Accepted: 16 December 2021 Published: 2 January 2022

Publisher's Note: MDPI stays neutral with regard to jurisdictional claims in published maps and institutional affiliations.

Copyright: (c) 2022 by the authors. Licensee MDPI, Basel, Switzerland. This article is an open access article distributed under the terms and conditions of the Creative Commons Attribution (CC BY) license (https:// creativecommons.org/licenses/by/ $4.0 /)$.

\begin{abstract}
The objective of the research article is two-fold. Firstly, we present a fixed point result in the context of triple controlled metric type spaces with a distinctive contractive condition involving the controlled functions. Secondly, we consider an initial value problem associated with a nonlinear Volterra-Fredholm integro-dynamic equation and examine the existence and uniqueness of solutions via fixed point theorem in the setting of complete triple controlled metric type spaces. Furthermore, the theorem is applied to illustrate the existence of a unique solution to an integro-dynamic equation.
\end{abstract}

Keywords: triple controlled metric type spaces; controlled functions; fixed point; Volterra-Fredholm integro-dynamic equations; time scales

MSC: 47H10; 45B05

\section{Introduction}

In science and technology, the solution of integral and integro-differential equations plays a significant role. A differential equation, an integral equation, or an integro-differential equation is obtained whenever a physical system is modeled in the differential context. On the other hand, Hilger [1] first introduced a time scale (or a measure chain) in 1988. Several researchers have built on different perspectives of the theory as Hilger developed the concepts of a derivative and integral on a time scale [2-6]. It has been demonstrated that time scales can be applied to any area that can be represented using discrete or continuous models.

Fixed point theory provides a basis in solving existence and uniqueness problems involving all types of differential and integral equations. Many researchers have examined the question of the existence and uniqueness of integrodifferential equations (see [7-16]). Recently, the authors in [17] discussed a particular kind of integro-dynamic equation.

Fixed point theory, on the other hand, is a significant idea with multiple applications in diverse fields of mathematics. The existence of fixed points has broad implications in a variety of disciplines of analysis and topology. It has its own implications and has progressed immensely over the last one and half centuries.

Banach's contraction principle, however, in the case of a metric space setting, is the basis of metric fixed point theory. Banach's contraction principle is a very useful tool in nonlinear analysis since it is an easy and flexible tool for defining existence and uniqueness theorems for operator equations. This fact prompted researchers to seek to expand and extrapolate it in order to broaden its scope of application as far as possible.

In recent times, we have seen a number of generalized metric spaces, such as extended $b$-metric spaces [18], controlled metric type spaces [19], double-controlled metric type spaces [20], controlled $b$-Branciari metric type spaces [21], triple controlled metric type 
spaces [22], and extended hexagonal $b$-metric spaces [23]; several authors concentrated their attention in order to acquire fixed point theorems in these kinds of spaces.

Although only a few investigations have been carried out on the existence and uniqueness of solutions of integro-dynamic equations, the main purpose of this research is to discuss the existence and uniqueness of Volterra-Fredholm integro-dynamic equations on time scales. We analyze the problem in the framework of complete triple controlled metric type spaces and apply a fixed point theorem with a contractive condition involving the controlled functions.

\section{Preliminaries}

We begin with a brief overview of the fundamental concepts of time scales.

Definition $1([4,7]) .1$. A time scale is an arbitrary non-empty closed subset of the real numbers. A time scale is usually denoted by the symbol $\mathbb{T}$.

2. For $t \in \mathbb{T}$, the forward jump operator $\sigma: \mathbb{T} \rightarrow \mathbb{T}$ is defined as

$$
\sigma(t)=\inf \{s \in \mathbb{T}: s>t\} .
$$

3. For $t \in \mathbb{T}$, the backward jump operator $\rho: \mathbb{T} \rightarrow \mathbb{T}$ is defined as

$$
\rho(t)=\sup \{s \in \mathbb{T}: s<t\} .
$$

4. We set

$$
\inf \varnothing=\sup \mathbb{T}, \sup \varnothing=\inf \mathbb{T} .
$$

Remark 1 ([17]). It is easy to see that, for any $t \in \mathbb{T}$, we have $\sigma(t) \geq t$ and $\rho(t) \leq t$.

Definition $2([4,7])$. We define the set $\mathbb{T}^{K}$ as

$$
\mathbb{T}^{K}=\left\{\begin{array}{l}
\mathbb{T} \backslash(\rho(\sup \mathbb{T}), \sup \mathbb{T}], \text { if } \sup \mathbb{T}<\infty \\
\mathbb{T}, \text { otherwise }
\end{array}\right.
$$

Definition 3 ([4,7]). Let $g: \mathbb{T} \rightarrow \mathbb{R}$ be a function and let $t \in \mathbb{T}^{K}$. We define $g^{\Delta}(t)$ to be the number, provided that it exists, as follows: for any $\epsilon>0$, there is a neighborhood $U$ of $t, U=(t-\delta$, $t+\delta) \cap \mathbb{T}$ for some $\delta>0$, such that

$$
\left|g(\sigma(t))-g(s)-g^{\Delta}(t)(\sigma(t)-s)\right| \leq \epsilon|\sigma(t)-s| \text { for all } s \in U, s \neq \sigma(t)-s .
$$

$g^{\Delta}(t)$ is called the delta or Hilger derivative of $g$ at $t$.

$g$ is the delta or Hilger differentiable or, in brielf, differentiable in $\mathbb{T}^{K}$ if $g^{\Delta}(t)$ exists for all $t \in \mathbb{T}^{K}$.

The function $g^{\Delta}: \mathbb{T} \rightarrow \mathbb{R}$ is said to be the delta derivative or Hilger derivative or, in brief, the derivative, of $g$ in $\mathbb{T}^{K}$.

Remark 2 ([17]). If $\mathbb{T}=\mathbb{R}$, then the delta derivative coincides with the classical derivative. Note that the delta derivative is well-defined.

It is worth noting that the nabla derivative, which is given in [7], is another form of derivative defined on time scales. We suggest some recent research upon these two types of fractional-order derivatives in $[4,7,24]$. 
Definition $4([4,7])$. A function $G: \mathbb{T} \rightarrow \mathbb{R}$ is called a delta antiderivative of $g: \mathbb{T} \rightarrow \mathbb{R}$ provided that $G^{\Delta}(t)=g(t)$ holds for all $t \in \mathbb{T}^{K}$. Then, the delta integral of $g$ is defined by

$$
\int_{a}^{b} g(t) \Delta t=G(b)-G(a) \text {. }
$$
ties.

Eventually, we define the monomials on time scales and consider a few of its proper-

Definition $5([4,5])$. The following is a recursive definition of monomials on time scales.

$$
\begin{aligned}
& h_{0}(t, \alpha)=1, \\
& h_{1}(t, \alpha)=\int_{\alpha}^{t} h_{0}(t, \alpha) \Delta(t)=t-\alpha, \\
& h_{k}(t, \alpha)=\int_{\alpha}^{t} h_{k-1}(t, \alpha) \Delta(t),
\end{aligned}
$$

for $k \geq 2$.

Theorem 1 ([5]). For each $k \in \mathbb{N}_{0}$, the inequality

$$
h_{k}(t, \alpha) \leq \frac{(t-\alpha)^{k}}{k !}
$$

holds for each $t, \alpha \in \mathbb{T}, t \geq \alpha$.

On the other hand, M. Frechet [25] developed the well-known conception of metric space as an outgrowth of conventional distance. In the literature, the notion of metric space is enlarged in a number of different ways (for instance, see [26-28]). The definition of triple controlled metric type space is described below, which is used extensively in our main results.

Definition 6 ([22]). Let $X$ be a non-empty set and $R, S, T: X \times X \rightarrow[1, \infty)$. A function $d_{\mathrm{T}}: X \times X \rightarrow[0, \infty)$ is called a triple controlled metric type if it satisfies:

(1) $d_{\mathrm{T}}(w, v)=0$ if and only if $w=v$ for all $w, v \in X$;

(2) $d_{\mathrm{T}}(w, v)=d_{\mathrm{T}}(v, w)$ for all $w, v \in X$;

(3) $d_{\mathrm{T}}(w, v) \leq R(w, e) d_{\mathrm{T}}(w, e)+S(e, f) d_{\mathrm{T}}(e, f)+T(f, v) d_{\mathrm{T}}(f, v)$

for all $w, v \in X$ and for all distinct points $e, f \in X$, each distinct from $w$ and $v$, respectively. The pair $\left(X, d_{\mathrm{T}}\right)$ is called a triple controlled metric type space (in short, TCMTS).

The extension of the rectangular inequality is the most important feature of triple controlled metric type spaces. The recent research pertaining to TCMTS can be found at $[22,29-31]$.

Example 1 ([22]). Let $X=P \cup Q$ where $P=\left\{\frac{1}{n}: n \in \mathbb{N}\right\}$ and $Q$ is the set of all positive integers. Define $d_{\mathrm{T}}: X \times X \rightarrow[0, \infty)$ such that ' $d_{\mathrm{T}}$ ' is symmetric and, for all $w, v \in X$

$$
d_{\mathrm{T}}(w, v)=\left\{\begin{array}{l}
0, \text { if } w=v \\
w+8, \text { if } w \in P, v \in\{6,7\} \text { or } w \in\{6,7\}, v \in P \\
2, \text { otherwise }
\end{array}\right.
$$


Let $R, S, T: X \times X \rightarrow[1, \infty)$ be defined as

$$
\begin{gathered}
R(w, v)=\left\{\begin{array}{l}
\frac{1}{w}, \text { if } w \in P, v \in Q \\
1, \text { otherwise }
\end{array}\right. \\
S(w, v)=\left\{\begin{array}{l}
w+v, \text { if } w \in P, v \in Q \\
2, \text { otherwise }
\end{array}\right.
\end{gathered}
$$

and

$$
T(w, v)=\left\{\begin{array}{l}
w+1, \text { if both } w, v \in P \text { or } w, v \in Q \\
\frac{3}{2}, \text { otherwise }
\end{array}\right.
$$

Therefore, $\left(X, d_{\mathrm{T}}\right)$ is a TCMTS.

Remark 3. By employing the same function(s), a triple controlled metric type becomes a controlled b-Branciari metric type space. In fact, the converse is not true (see Example 3.2 in [22]).

Contractive mappings have aroused renewed interest, known for their ability to reduce the number of iterations required when working with numerical calculations of fixed point type problems. In the next section, we establish a fixed point theorem in the context of TCMTS under a new contractive condition employing the controlled functions.

\section{Fixed Point Theorem}

Theorem 2. Let $\left(X, d_{\mathrm{T}}\right)$ be a complete triple controlled metric type space and $A: X \rightarrow X$ be a self mapping on $X$. Assume that $R(w, v) S(w, v)>T(w, v)$ for any $w, v \in X$ and there exists $\beta \in[0,1)$ such that

$$
d_{\mathrm{T}}(A w, A v) \leq \beta \frac{R(w, v) S(w, v)}{T(w, v)} d_{\mathrm{T}}(w, v), \text { for all } w, v \in X .
$$

For each $w_{0} \in X$ and $w_{n}=A^{n} w_{0}, n \in \mathbb{N}$, we have

$$
\sup _{m} \lim _{n} T\left(w_{n}, w_{m}\right) \frac{R\left(w_{n}, w_{n+1}\right) S\left(w_{n-1}, w_{n}\right)}{T\left(w_{n-1}, w_{n}\right)}<\frac{1}{\beta^{2}}
$$

and

$$
\sup _{m} \lim _{n} T\left(w_{n}, w_{m}\right) \frac{R\left(w_{n}, w_{n+1}\right) S\left(w_{n+1}, w_{n+2}\right)}{T\left(w_{n}, w_{n+1}\right)}<\frac{1}{\beta^{2}}
$$

then, $w_{n} \rightarrow$ was $n \rightarrow \infty$. Moreover, if

$$
\lim _{n \rightarrow+\infty} \frac{R\left(w, w_{n}\right) S\left(w, w_{n}\right)}{T\left(w, w_{n}\right)} \text { and } \lim _{n \rightarrow+\infty} \frac{R\left(w_{n}, w\right) S\left(w_{n}, w\right)}{T\left(w_{n}, w\right)}
$$

exist and are finite, then $A$ has a fixed point in X. Furthermore, $A$ has a unique fixed point if

$$
\frac{R(A w, A v) S(A w, A v)}{T(A w, A v)}<\frac{1}{\beta^{\prime}}
$$

for any two fixed points $w, v \in X$ of $A$.

Proof. Let $w_{0} \in X$ and define an iterative sequence $\left\{w_{n}\right\}$ by

$$
w_{0}, A w_{0}=w_{1}, A w_{1}=w_{2} \Rightarrow w_{2}=A^{2} w_{0}, \ldots, w_{n+1}=A^{n+1} w_{0} .
$$


Consider

$$
\begin{aligned}
d_{T}\left(w_{n}, w_{n+1}\right)= & d_{T}\left(A w_{n-1}, A w_{n}\right) \\
\leq & \beta \frac{R\left(w_{n-1}, w_{n}\right) S\left(w_{n-1}, w_{n}\right)}{T\left(w_{n-1}, w_{n}\right)} d_{T}\left(w_{n-1}, w_{n}\right) \\
& \vdots \\
\leq & \beta^{n} \prod_{l=1}^{n} \frac{R\left(w_{l-1}, w_{l}\right) S\left(w_{l-1}, w_{l}\right)}{T\left(w_{l-1}, w_{l}\right)} d_{T}\left(w_{0}, w_{1}\right) .
\end{aligned}
$$

By using a similar method, we obtain

$$
d_{T}\left(w_{n}, w_{n+2}\right) \leq \beta^{n} \prod_{l=1}^{n} \frac{R\left(w_{l-1}, w_{l+1}\right) S\left(w_{l-1}, w_{l+1}\right)}{T\left(w_{l-1}, w_{l+1}\right)} d_{T}\left(w_{0}, w_{2}\right) .
$$

It is indeed significant to note that if we take the limit of each of the inequalities above as $n \rightarrow \infty$, we obtain

$$
\lim _{n \rightarrow \infty} d_{T}\left(w_{n}, w_{n+1}\right)=\lim _{n \rightarrow \infty} d_{T}\left(w_{n}, w_{n+2}\right)=0 .
$$

If $w_{n}=w_{m}$ for some $n \neq m$, i.e., for $m>n$, we have $A^{m-n}\left(w_{n}\right)=w_{n}$. Choose $v=w_{n}$ and $q=m-n$, and then we obtain $A^{q} v=v$, i.e., $v$ is a periodic point of $A$. Thus,

$$
d_{T}(v, A v)=d_{T}\left(A^{q} v, A^{q+1} v\right) \leq \beta^{q} \prod_{l=1}^{q} \frac{R\left(A^{l-1} w, A^{l} w\right) S\left(A^{l-1} w, A^{l} w\right)}{T\left(A^{l-1} w, A^{l} w\right)} d_{T}(w, A w) .
$$

As $\beta \in(0,1)$, we obtain $d_{T}(v, A v)=0$. Hence, $v$ is a fixed point of $A$.

Assume that $w_{n} \neq w_{m}$ for some $n \neq m$. To verify that $\left\{w_{n}\right\}$ is a Cauchy sequence, we need to show $\lim _{n, m \rightarrow \infty} d_{T}\left(w_{n}, w_{m}\right)=0$. Consider $d_{T}\left(w_{n}, w_{m}\right)$ with odd $m$; we obtain the desired result by continuously applying the controlled rectangular inequality.

$$
\begin{aligned}
d_{T}\left(w_{n}, w_{m}\right) \leq & R\left(w_{n}, w_{n+1}\right) d_{T}\left(w_{n}, w_{n+1}\right)+S\left(w_{n+1}, w_{n+2}\right) d_{T}\left(w_{n+1}, w_{n+2}\right) \\
& +T\left(w_{n+2}, w_{m}\right) d_{T}\left(w_{n+2}, w_{m}\right) \\
& \vdots \\
\leq & R\left(w_{n}, w_{n+1}\right) d_{T}\left(w_{n}, w_{n+1}\right)+S\left(w_{n+1}, w_{n+2}\right) d_{T}\left(w_{n+1}, w_{n+2}\right)+ \\
& \sum_{i=\frac{n}{2}+1}^{\frac{m-1}{2}} R\left(w_{2 i}, w_{2 i+1}\right) d_{T}\left(w_{2 i}, w_{2 i+1}\right) \prod_{j=\frac{n}{2}+1}^{i} T\left(w_{2 j}, w_{m}\right)+ \\
& \sum_{i=\frac{n}{2}+1}^{\frac{m-3}{2}} S\left(w_{2 i+1}, w_{2 i+2}\right) d_{T}\left(w_{2 i+1}, w_{2 i+2}\right) \prod_{j=\frac{n}{2}+1}^{i} T\left(w_{2 j}, w_{m}\right) \\
\leq & \sum_{i=\frac{n}{2}}^{\frac{m-1}{2}} R\left(w_{2 i}, w_{2 i+1}\right) d_{T}\left(w_{2 i}, w_{2 i+1}\right) \prod_{j=\frac{n}{2}}^{i} T\left(w_{2 j}, w_{m}\right)+ \\
& \sum_{i=\frac{n}{2}} \frac{m-3}{2} S\left(w_{2 i+1}, w_{2 i+2}\right) d_{T}\left(w_{2 i+1}, w_{2 i+2}\right) \prod_{j=\frac{n}{2}}^{i} T\left(w_{2 j}, w_{m}\right) \\
\leq & \sum_{i=\frac{n}{2}}^{\frac{m-1}{2}} R\left(w_{2 i}, w_{2 i+1}\right) \prod_{j=\frac{n}{2}}^{i} T\left(w_{2 j}, w_{m}\right) \beta^{2 i} \prod_{l=1}^{2 i} \frac{R\left(w_{l-1}, w_{l}\right) S\left(w_{l-1}, w_{l}\right)}{T\left(w_{l-1}, w_{l}\right)} d_{T}\left(w_{0}, w_{1}\right)+ \\
& \sum_{i=\frac{n}{2}}^{\frac{m-3}{2}} S\left(w_{2 i+1}, w_{2 i+2}\right) \prod_{j=\frac{n}{2}}^{i} T\left(w_{2 j}, w_{m}\right) \beta^{2 i+1} \prod_{l=1}^{2 i+1} \frac{R\left(w_{l-1}, w_{l}\right) S\left(w_{l-1}, w_{l}\right)}{T\left(w_{l-1}, w_{l}\right)} d_{T}\left(w_{0}, w_{1}\right) .
\end{aligned}
$$


Let

$$
a_{i}=\prod_{j=\frac{n}{2}}^{i} T\left(w_{2 j}, w_{m}\right) \beta^{2 i} \prod_{l=1}^{2 i+1} R\left(w_{l-1}, w_{l}\right) \prod_{l=1}^{2 i} \frac{S\left(w_{l-1}, w_{l}\right)}{T\left(w_{l-1}, w_{l}\right)} d_{T}\left(w_{0}, w_{1}\right)
$$

and

$$
b_{i}=\prod_{j=\frac{n}{2}}^{i} T\left(w_{2 j}, w_{m}\right) \beta^{2 i+1} \prod_{l=1}^{2 i+1} \frac{R\left(w_{l-1}, w_{l}\right)}{T\left(w_{l-1}, w_{l}\right)} \prod_{l=1}^{2 i+2} S\left(w_{l-1}, w_{l}\right) d_{T}\left(w_{0}, w_{1}\right) .
$$

Through using Equation (4) and Equation (5), we obtain

$$
\sup _{m \geq 1} \lim _{i} \frac{a_{i+1}}{a_{i}}=\sup _{m \geq 1} \lim _{i} \beta^{2} T\left(w_{2 i+2}, w_{m}\right) \frac{R\left(w_{2 i+2}, w_{2 i+3}\right) S\left(w_{2 i+1}, w_{2 i+2}\right)}{T\left(w_{2 i+1}, w_{2 i+2}\right)}<1
$$

and

$$
\sup _{m \geq 1} \lim _{i} \frac{b_{i+1}}{b_{i}}=\sup _{m \geq 1} \lim _{i} \beta^{2} T\left(w_{2 i+2}, w_{m}\right) \frac{R\left(w_{2 i+2}, w_{2 i+3}\right) S\left(w_{2 i+3}, w_{2 i+4}\right)}{T\left(w_{2 i+2}, w_{2 i+3}\right)}<1 .
$$

Thereby, we infer

$$
\sum_{i=\frac{n}{2}}^{+\infty} \beta^{2 i} \prod_{j=\frac{n}{2}}^{i} T\left(w_{2 j}, w_{m}\right) \prod_{l=1}^{2 i+1} R\left(w_{l-1}, w_{l}\right) \prod_{l=1}^{2 i} \frac{S\left(w_{l-1}, w_{l}\right)}{T\left(w_{l-1}, w_{l}\right)} d_{T}\left(w_{0}, w_{1}\right)<+\infty
$$

and

$$
\sum_{i=\frac{n}{2}}^{+\infty} \beta^{2 i+1} \prod_{j=\frac{n}{2}}^{i} T\left(w_{2 j}, w_{m}\right) \prod_{l=1}^{2 i+1} \frac{R\left(w_{l-1}, w_{l}\right)}{T\left(w_{l-1}, w_{l}\right)} \prod_{l=1}^{2 i+2} S\left(w_{l-1}, w_{l}\right) d_{T}\left(w_{0}, w_{1}\right)<+\infty .
$$

Consequently,

$$
\left(\sum_{i=\frac{n}{2}}^{\frac{m-1}{2}} \beta^{2 i} \prod_{j=\frac{n}{2}}^{i} T\left(w_{2 j}, w_{m}\right) \prod_{l=1}^{2 i+1} R\left(w_{l-1}, w_{l}\right) \prod_{l=1}^{2 i} \frac{S\left(w_{l-1}, w_{l}\right)}{T\left(w_{l-1}, w_{l}\right)} d_{T}\left(w_{0}, w_{1}\right)\right)
$$

and

$$
\left(\sum_{i=\frac{n}{2}}^{\frac{m-3}{2}} \beta^{2 i+1} \prod_{j=\frac{n}{2}}^{i} T\left(w_{2 j}, w_{m}\right) \prod_{l=1}^{2 i+1} \frac{R\left(w_{l-1}, w_{l}\right)}{T\left(w_{l-1}, w_{l}\right)} \prod_{l=1}^{2 i+2} S\left(w_{l-1}, w_{l}\right) d_{T}\left(w_{0}, w_{1}\right)\right)
$$

are Cauchy sequences in $\mathbb{R}$. Hence, $\lim _{n, m \rightarrow \infty} d_{T}\left(w_{n}, w_{m}\right)=0$, for odd $m$.

From the other end, if we consider $d_{T}\left(w_{n}, w_{m}\right)$ with even $m$, and applying controlled rectangular inequality continuously, we obtain

$$
\begin{aligned}
d_{T}\left(w_{n}, w_{m}\right) \leq & \sum_{i=\frac{n}{2}}^{\frac{m-4}{2}}\left[R\left(w_{2 i}, w_{2 i+1}\right) d_{T}\left(w_{2 i}, w_{2 i+1}\right)+S\left(w_{2 i+1}, w_{2 i+2}\right) d_{T}\left(w_{2 i+1}, w_{2 i+2}\right)\right] \\
& \times \prod_{j=\frac{n}{2}}^{i} T\left(w_{2 j}, w_{m}\right)+\prod_{i=\frac{n}{2}+1}^{\frac{m-2}{2}} R\left(w_{2 i}, w_{m}\right) d_{T}\left(w_{m-2}, w_{m}\right) \\
\leq & \sum_{i=\frac{n}{2}}^{\frac{m-4}{2}} R\left(w_{2 i}, w_{2 i+1}\right) \prod_{j=\frac{n}{2}}^{i} T\left(w_{2 j}, w_{m}\right) \beta^{2 i} \prod_{l=1}^{2 i} \frac{R\left(w_{l-1}, w_{l}\right) S\left(w_{l-1}, w_{l}\right)}{T\left(w_{l-1}, w_{l}\right)} d_{T}\left(w_{0}, w_{1}\right)
\end{aligned}
$$




$$
\begin{aligned}
& +\sum_{i=\frac{n}{2}}^{\frac{m-4}{2}} S\left(w_{2 i+1}, w_{2 i+2}\right) \prod_{j=\frac{n}{2}}^{i} T\left(w_{2 j}, w_{m}\right) \beta^{2 i+1} \prod_{l=1}^{2 i+1} \frac{R\left(w_{l-1}, w_{l}\right) S\left(w_{l-1}, w_{l}\right)}{T\left(w_{l-1}, w_{l}\right)} d_{T}\left(w_{0}, w_{1}\right) \\
& +\prod_{i=\frac{n}{2}+1}^{\frac{m-2}{2}} R\left(w_{2 i}, w_{m}\right) \beta^{m-2} \prod_{l=1}^{m-2} \frac{R\left(w_{l-1}, w_{l+1}\right) S\left(w_{l-1}, w_{l+1}\right)}{T\left(w_{l-1}, w_{l+1}\right)} d_{T}\left(w_{0}, w_{2}\right) .
\end{aligned}
$$

By following the procedure above, we can deduce that

$$
\left(\sum_{i=\frac{n}{2}}^{\frac{m-4}{2}} \beta^{2 i} \prod_{j=\frac{n}{2}}^{i} T\left(w_{2 j}, w_{m}\right) \prod_{l=1}^{2 i+1} R\left(w_{l-1}, w_{l}\right) \prod_{l=1}^{2 i} \frac{S\left(w_{l-1}, w_{l}\right)}{T\left(w_{l-1}, w_{l}\right)} d_{T}\left(w_{0}, w_{1}\right)\right)
$$

and

$$
\left(\sum_{i=\frac{n}{2}}^{\frac{m-4}{2}} \beta^{2 i+1} \prod_{j=\frac{n}{2}}^{i} T\left(w_{2 j}, w_{m}\right) \prod_{l=1}^{2 i+1} \frac{R\left(w_{l-1}, w_{l}\right)}{T\left(w_{l-1}, w_{l}\right)} \prod_{l=1}^{2 i+2} S\left(w_{l-1}, w_{l}\right) d_{T}\left(w_{0}, w_{1}\right)\right)
$$

are Cauchy sequences in $\mathbb{R}$. Therefore, we conclude that $\lim _{n, m \rightarrow \infty} d_{T}\left(w_{n}, w_{m}\right)=0$, for even $m$. Hence, in all the cases, we obtain

$$
d_{T}\left(w_{n}, w_{m}\right) \rightarrow 0 \text { as } n, m \rightarrow \infty
$$

i.e., $\left\{w_{n}\right\}$ is a Cauchy sequence in $X$. Therefore, by the above equation, as well as the completeness property of $\left(X, d_{T}\right)$, we obtain $\lim _{n \rightarrow+\infty} w_{n}=w$ for $w \in X$. We will now illustrate that $w$ is a fixed point of $A$. From inequalities (6) and (12), it is simple to prove

$$
\lim _{n \rightarrow+\infty} d_{T}\left(w_{n+2}, w\right)=0 .
$$

Consider

$$
\begin{aligned}
d_{T}(A w, w) \leq & R\left(A w, w_{n+1}\right) d_{T}\left(A w, w_{n+1}\right)+S\left(w_{n+1}, w_{n+2}\right) d_{T}\left(w_{n+1}, w_{n+2}\right)+T\left(w_{n+2}, w\right) d_{T}\left(w_{n+2}, w\right) \\
= & R\left(A w, A w_{n}\right) d_{T}\left(A w, A w_{n}\right)+S\left(w_{n+1}, w_{n+2}\right) d_{T}\left(w_{n+1}, w_{n+2}\right)+T\left(w_{n+2}, w\right) d_{T}\left(w_{n+2}, w\right) \\
\leq & R\left(A w, A w_{n}\right) \beta \frac{R\left(w, w_{n}\right) S\left(w, w_{n}\right)}{T\left(w, w_{n}\right)} d_{T}\left(w, w_{n}\right)+S\left(w_{n+1}, w_{n+2}\right) d_{T}\left(w_{n+1}, w_{n+2}\right) \\
& +T\left(w_{n+2}, w\right) d_{T}\left(w_{n+2}, w\right) .
\end{aligned}
$$

By Equation (13) and the assumption of the theorem, we obtain $d_{T}(A w, w) \leq 0$ as $n \rightarrow$ $\infty$. Therefore, $d_{T}(A w, w)=0$, i.e., $A w=w$. Hence, $w$ is a fixed point of $A$.

Finally, we show the uniqueness of the fixed point. Let $w, v$ be two distinct fixed points of $A$; then, $A w=w$ and $A v=v$. By employing the inequality (7), one can obtain

$$
d_{T}(w, v)=d_{T}(A w, A v) \leq \beta \frac{R(w, v) S(w, v)}{T(w, v)} d_{T}(w, v)<d_{T}(w, v),
$$

which is a contradiction. Thereby, $w$ is a unique fixed point of $A$.

One of the most significant applications of our result is to prove the existence and uniqueness of the Volterra-Fredholm integro-dynamic equation of the second kind, which is defined below.

\section{Volterra-Fredholm Integro-Dynamic Equation of Second Kind}

Let $\mathbb{T}$ be a time scale with delta differential operator $\Delta$ and forward jump operator $\sigma$, respectively.

Definition 7. A Volterra-Fredholm integro-dynamic equation of the second kind is given as 


$$
\begin{aligned}
& u^{\Delta^{n}}(w)=h(w)+ \int_{w_{0}}^{w} C(s, w, \sigma(s), \sigma(w), u(s)) \Delta s+\int_{w_{0}}^{v_{0}} D(s, w, \sigma(s), \sigma(w), u(s)) \Delta s, w \in\left[w_{0}, v_{0}\right]_{\mathbb{T}} \\
& \begin{aligned}
\text { where } h:\left[w_{0}, v_{0}\right]_{\mathbb{T}} \rightarrow \mathbb{R} \text { and } C, D:\left(\left[w_{0}, v_{0}\right]_{\mathbb{T}}\right)^{4} \times \mathbb{R} \rightarrow \mathbb{R} \text { are given functions, and } u \text { is the } \\
\text { unknown function. }
\end{aligned} \\
& \quad \text { In this work, we deal with the case where Equation (14) has a first-order } \Delta \text {-derivative, } \\
& \text { i.e., an equation of the type } \\
& u^{\Delta}(w)=h(w)+\int_{w_{0}}^{w} C(s, w, \sigma(s), \sigma(w), u(s)) \Delta s+\int_{w_{0}}^{v_{0}} D(s, w, \sigma(s), \sigma(w), u(s)) \Delta s, w \in\left[w_{0}, v_{0}\right]_{\mathbb{T}} .
\end{aligned}
$$

\section{Existence and Uniqueness Theorem}

In this segment, we focus on an initial value problem concerned with the nonlinear Volterra-Fredholm integro-dynamic equations and examine whether its solution exists and is unique in the TCMTS setting.

Let $\mathbb{T}$ be a time scale with delta differential operator $\Delta$ and forward jump operator $\sigma$, respectively. Consider the initial value problem

$$
\begin{aligned}
u^{\Delta}(w)=h(w) & +\int_{w_{0}}^{w} K_{1}(s, w, \sigma(s), \sigma(w)) F(u(s)) \Delta s \\
& +\int_{w_{0}}^{v_{0}} K_{2}(s, w, \sigma(s), \sigma(w)) G(u(s)) \Delta s, w \in\left[w_{0}, v_{0}\right]_{\mathbb{T}} \\
u\left(w_{0}\right)=a, &
\end{aligned}
$$

where $h:\left[w_{0}, v_{0}\right]_{\mathbb{T}} \rightarrow \mathbb{R}, K_{1}, K_{2}:\left(\left[w_{0}, v_{0}\right]_{\mathbb{T}}\right)^{4} \rightarrow \mathbb{R}, F, G: \mathbb{R} \rightarrow \mathbb{R}$ are given functions. Let $C\left[w_{0}, v_{0}\right]_{\mathbb{T}}$ be the space of continuous functions on $\left[w_{0}, v_{0}\right]_{\mathbb{T}}$ and $d_{T}: C\left[w_{0}, v_{0}\right]_{\mathbb{T}} \times$ $C\left[w_{0}, v_{0}\right]_{\mathbb{T}} \rightarrow[0, \infty)$ be defined as

$$
d_{T}(w, v)=\sup _{t \in\left[w_{0}, v_{0}\right]_{\mathbb{T}}}|w(t)-v(t)|^{2}
$$

Consequently, $\left(C\left[w_{0}, v_{0}\right]_{\mathbb{T}}, d_{T}\right)$ is a complete triple controlled metric space with the below given controlled functions:

$$
\begin{gathered}
R(w, v)=\left\{\begin{array}{l}
66+23 \sup _{t \in\left[w_{0}, v_{0}\right]_{\mathbb{T}}}|w(t)-v(t)|^{2}, \text { if } w(t) \neq v(t) \\
1, \text { if } w(t)=v(t)
\end{array}\right. \\
S(w, v)=\left\{\begin{array}{l}
\sup _{t \in\left[w_{0}, v_{0}\right]_{\mathbb{T}}}\left(\left(1+|w(t)|^{2}\right)\left(1+|v(t)|^{2}\right)\right), \text { if } w(t) \neq v(t) \\
1, \text { if } w(t)=v(t)
\end{array}\right. \\
T(w, v)=\left\{\begin{array}{l}
1+\sup _{t \in\left[w_{0}, v_{0}\right]_{\mathbb{T}}}|w(t)-v(t)|^{2}, \text { if } w(t) \neq v(t) \\
1, \text { if } w(t)=v(t)
\end{array}\right.
\end{gathered}
$$

Firstly, we reveal that the initial value problem specified in Equation (16) can be rewritten as

$$
\begin{aligned}
u(t)=a & +\int_{w_{0}}^{t} h(w) \Delta w+\int_{w_{0}}^{t} \int_{w_{0}}^{w} K_{1}(s, w, \sigma(s), \sigma(w)) F(u(s)) \Delta s \Delta w \\
& +\int_{w_{0}}^{t} \int_{w_{0}}^{v_{0}} K_{2}(s, w, \sigma(s), \sigma(w)) G(u(s)) \Delta s \Delta w, \quad t \in\left[w_{0}, v_{0}\right]_{\mathbb{T}}
\end{aligned}
$$


when both sides of $\left[w_{0}, t\right]$ have their delta integrals taken, where $t \in\left[w_{0}, v_{0}\right]_{\mathbb{T}}$. It is evident that if the functions $h, K_{1}, K_{2}, F$ and $G$ are delta integrable, then the right-hand side of Equation (18) is a continuous function on $\left[w_{0}, v_{0}\right]_{\mathbb{T}}$. Define the mapping $A: C\left[w_{0}, v_{0}\right]_{\mathbb{T}} \rightarrow$ $C\left[w_{0}, v_{0}\right]_{\mathbb{T}}$ as

$$
\begin{aligned}
A u(t)= & +\int_{w_{0}}^{t} h(w) \Delta w+\int_{w_{0}}^{t} \int_{w_{0}}^{w} K_{1}(s, w, \sigma(s), \sigma(w)) F(u(s)) \Delta s \Delta w \\
& +\int_{w_{0}}^{t} \int_{w_{0}}^{v_{0}} K_{2}(s, w, \sigma(s), \sigma(w)) G(u(s)) \Delta s \Delta w, \quad t \in\left[w_{0}, v_{0}\right]_{\mathbb{T}} .
\end{aligned}
$$

A solution to the problem mentioned in Equation (16) (equivalently Equation (18)) is, evidently, a fixed point of $A$. The existence uniqueness theorem for the solution of Equation (16) is presented in the following section.

Theorem 3. Let $\mathbb{T}$ be a time scale and $\left[w_{0}, v_{0}\right]_{\mathbb{T}}$ be a finite interval for some $w_{0}, v_{0} \in \mathbb{T}$. Assume that the following conditions are satisfied:

1. The functions $h, K_{1}$ and $K_{2}$ are delta integrable on $\left[w_{0}, v_{0}\right]_{\mathbb{T}}$.

2. The functions $F$ and $G$ are delta integrable on $\left[w_{0}, v_{0}\right]_{\mathbb{T}}$ and satisfy

$$
\begin{aligned}
& \text { (i) }|F(u(s))-F(z(s))| \leq \sqrt{66 \beta}|u(s)-z(s)| \frac{|u(s)||z(s)|}{\sqrt{1+|u(s)-z(s)|^{2}}}, \\
& \text { (ii) }|G(u(s))-G(z(s))| \leq \sqrt{23 \beta} \frac{|u(s)-z(s)|^{\frac{5}{2}}}{\sqrt{1+|u(s)-z(s)|^{2}}}
\end{aligned}
$$

for some $\beta \in\left[0, \frac{1}{178\left(1+\left[a+\sup _{t}|h(t)|+\sup _{t, s}\left|K_{1}(s, t, \sigma(s), \sigma(t))\right||F(u(s))|+\sup _{t, s}\left|K_{2}(s, t, \sigma(s), \sigma(t))\right||G(u(s))|\right]^{2}\right)^{6}}\right)$.

3.

$$
\begin{aligned}
& \text { (i) } \int_{w_{0}}^{t} \int_{w_{0}}^{w}\left[K_{1}(s, w, \sigma(s), \sigma(w))\right]^{2} \Delta s \Delta w \leq F_{1}, \\
& \text { (ii) } \int_{w_{0}}^{t} \int_{w_{0}}^{v_{0}}\left[K_{2}(s, w, \sigma(s), \sigma(w))\right]^{2} \Delta s \Delta w \leq F_{2}, w, t \in\left[w_{0}, v_{0}\right]_{\mathbb{T}}
\end{aligned}
$$

for some $F_{1}<\frac{1}{\left(t-w_{0}\right)^{2}}, \quad F_{2}<\frac{1}{2\left(v_{0}-w_{0}\right)^{2}}$.

4.

(i) $F\left(a+\int_{w_{0}}^{t} h(w) \Delta w+\int_{w_{0}}^{t} \int_{w_{0}}^{w} K_{1}(s, w, \sigma(s), \sigma(w)) F(u(s)) \Delta s \Delta w\right.$

$$
\left.+\int_{w_{0}}^{t} \int_{w_{0}}^{v_{0}} K_{2}(s, w, \sigma(s), \sigma(w)) G(u(s)) \Delta s \Delta w\right)<F(u(s)),
$$

(ii) $G\left(a+\int_{w_{0}}^{t} h(w) \Delta w+\int_{w_{0}}^{t} \int_{w_{0}}^{w} K_{1}(s, w, \sigma(s), \sigma(w)) F(u(s)) \Delta s \Delta w\right.$

$$
\left.+\int_{w_{0}}^{t} \int_{w_{0}}^{v_{0}} K_{2}(s, w, \sigma(s), \sigma(w)) G(u(s)) \Delta s \Delta w\right)<G(u(s)) .
$$

Hence, the map A specified in Equation (19) has a unique fixed point, implying that the integral equation in Equation (18) has a unique solution in $C\left[w_{0}, v_{0}\right]_{\mathbb{T}}$.

Proof. According to the definition of the map A defined in Equation (19) and the CauchySchwarz inequality for integrals on time scales [2], we have 


$$
\begin{aligned}
& |A u(t)-A z(t)|^{2}=(A u(t)-A z(t))^{2} \\
& =\left[\int_{w_{0}}^{t} \int_{w_{0}}^{w} K_{1}(s, w, \sigma(s), \sigma(w))[F(u(s))-F(z(s))] \Delta s \Delta w\right. \\
& \left.+\int_{w_{0}}^{t} \int_{w_{0}}^{v_{0}} K_{2}(s, w, \sigma(s), \sigma(w))[G(u(s))-G(z(s))] \Delta s \Delta w\right]^{2} \\
& \leq 2\left[\int_{w_{0}}^{t} \int_{w_{0}}^{w} K_{1}(s, w, \sigma(s), \sigma(w))[F(u(s))-F(z(s))] \Delta s \Delta w\right]^{2} \\
& +2\left[\int_{w_{0}}^{t} \int_{w_{0}}^{v_{0}} K_{2}(s, w, \sigma(s), \sigma(w))[G(u(s))-G(z(s))] \Delta s \Delta w\right]^{2} \\
& \leq 2\left(\int_{w_{0}}^{t} \int_{w_{0}}^{w}\left[K_{1}(s, w, \sigma(s), \sigma(w))\right]^{2} \Delta s \Delta w\right)\left(\int_{w_{0}}^{t} \int_{w_{0}}^{w}|F(u(s))-F(z(s))|^{2} \Delta s \Delta w\right) \\
& +2\left(\int_{w_{0}}^{t} \int_{w_{0}}^{v_{0}}\left[K_{2}(s, w, \sigma(s), \sigma(w))\right]^{2} \Delta s \Delta w\right)\left(\int_{w_{0}}^{t} \int_{w_{0}}^{v_{0}}|G(u(s))-G(z(s))|^{2} \Delta s \Delta w\right) \\
& \leq 2 F_{1} \int_{w_{0}}^{t} \int_{w_{0}}^{w} 66 \beta \frac{|u(s)-z(s)|^{2}|u(s)|^{2}|z(s)|^{2}}{1+|u(s)-z(s)|^{2}} \Delta s \Delta w \\
& +2 F_{2} \int_{w_{0}}^{t} \int_{w_{0}}^{v_{0}} 23 \beta \frac{|u(s)-z(s)|^{5}}{1+|u(s)-z(s)|^{2}} \Delta s \Delta w \\
& \leq 2 F_{1} \int_{w_{0}}^{t} \int_{w_{0}}^{w} 66 \beta \frac{|u(s)-z(s)|^{2}\left(1+|u(s)|^{2}\right)\left(1+|z(s)|^{2}\right)}{1+|u(s)-z(s)|^{2}} \Delta s \Delta w \\
& +2 F_{2} \int_{w_{0}}^{t} \int_{w_{0}}^{v_{0}} 23 \beta \frac{|u(s)-z(s)|^{4}\left(1+|u(s)|^{2}\right)\left(1+|z(s)|^{2}\right)}{1+|u(s)-z(s)|^{2}} \Delta s \Delta w .
\end{aligned}
$$

Taking the supremum over $\left[w_{0}, v_{0}\right]_{\mathbb{T}}$ and in accordance with the metric's definition given in Equation (17), we obtain

$d_{T}(A u, A z) \leq \beta d_{T}(u, z) \frac{\sup _{t}\left\{\left(1+|u(t)|^{2}\right)\left(1+|z(t)|^{2}\right)\right\}}{1+\sup _{t}|u(t)-z(t)|^{2}}\left[132 F_{1} \int_{w_{0}}^{t} \int_{w_{0}}^{w} \Delta s \Delta w+46 F_{2} \sup _{t}|u(t)-z(t)|^{2} \int_{w_{0}}^{t} \int_{w_{0}}^{v_{0}} \Delta s \Delta w\right]$

Notice that

$$
\begin{aligned}
F_{1} \int_{w_{0}}^{t} \int_{w_{0}}^{w} \Delta s \Delta w=F_{1} \int_{w_{0}}^{t}\left(w-w_{0}\right) \Delta w=F_{1} \int_{w_{0}}^{t} h_{1}\left(w, w_{0}\right) \Delta w & =F_{1} h_{2}\left(t, w_{0}\right) \\
& \leq F_{1} \frac{\left(t-w_{0}\right)^{2}}{2}<\frac{1}{2}
\end{aligned}
$$

and

$$
\begin{aligned}
F_{2} \int_{w_{0}}^{t} \int_{w_{0}}^{v_{0}} \Delta s \Delta w=F_{2} \int_{w_{0}}^{t}\left(v_{0}-w_{0}\right) \Delta w & =F_{2}\left(v_{0}-w_{0}\right)\left(t-w_{0}\right) \\
& <F_{2}\left(v_{0}-w_{0}\right)^{2}<\frac{1}{2}
\end{aligned}
$$


at which $h_{1}$ and $h_{2}$ are the time scale monomials described in Equation (1). Hence, Equation (22) can be modified as

$$
\begin{aligned}
d_{T}(A u, A z) & \leq \beta d_{T}(u, z) \frac{\sup _{t}\left\{\left(1+|u(t)|^{2}\right)\left(1+|z(t)|^{2}\right)\right\}}{1+\sup _{t}|u(t)-z(t)|^{2}}\left[66+23 \sup _{t}|u(t)-z(t)|^{2}\right] \\
& =\beta \frac{R(u, z) S(u, z)}{T(u, z)} d_{T}(u, z),
\end{aligned}
$$

where $R(u, z)=66+23 \sup _{t \in\left[w_{0}, v_{0}\right]_{\mathrm{T}}}|u(t)-z(t)|^{2}, S(u, z)=\sup _{t \in\left[w_{0}, v_{0}\right]_{\mathbb{T}}}\left\{\left(1+|u(t)|^{2}\right)\left(1+|z(t)|^{2}\right)\right\}$ and $T(u, z)=1+\sup _{t \in\left[w_{0}, v_{0}\right]_{\mathbb{T}}}|u(t)-z(t)|^{2}$, respectively. It is obvious that $R(u, z) S(u, z)>T(u, z)$, for all $u, z \in C\left[w_{0}, v_{0}\right]_{\mathbb{T}}$. Consider

$$
\begin{aligned}
\left(A^{n} u\right)(t)= & A\left(A^{n-1} u(t)\right) \\
= & +\int_{w_{0}}^{t} h(w) \Delta w+\int_{w_{0}}^{t} \int_{w_{0}}^{w} K_{1}(s, w, \sigma(s), \sigma(w)) F\left(A^{n-1} u(t)\right) \Delta s \Delta w \\
& +\int_{w_{0}}^{t} \int_{w_{0}}^{v_{0}} K_{2}(s, w, \sigma(s), \sigma(w)) G\left(A^{n-1} u(t)\right) \Delta s \Delta w \\
= & +\int_{w_{0}}^{t} h(w) \Delta w+\int_{w_{0}}^{t} \int_{w_{0}}^{w} K_{1}(s, w, \sigma(s), \sigma(w)) F\left(A\left(A^{n-2} u(t)\right)\right) \Delta s \Delta w \\
& +\int_{w_{0}}^{t} \int_{w_{0}}^{v_{0}} K_{2}(s, w, \sigma(s), \sigma(w)) G\left(A\left(A^{n-2} u(t)\right)\right) \Delta s \Delta w \\
= & +\int_{w_{0}}^{t} h(w) \Delta w \\
& +\int_{w_{0}}^{t} \int_{w_{0}}^{w} K_{1}(s, w, \sigma(s), \sigma(w)) F\left(a+\int_{w_{0}}^{t} h(w) \Delta w+\int_{w_{0}}^{t} \int_{w_{0}}^{w} K_{1}(s, w, \sigma(s), \sigma(w))\right. \\
& \left.F\left(A^{n-2} u(t)\right) \Delta s \Delta w+\int_{w_{0}}^{t} \int_{w_{0}}^{v_{0}} K_{2}(s, w, \sigma(s), \sigma(w)) G\left(A^{n-2} u(t)\right) \Delta s \Delta w\right) \Delta s \Delta w \\
& +\int_{w_{0}}^{t} \int_{w_{0}}^{v_{0}} K_{2}(s, w, \sigma(s), \sigma(w)) G\left(a+\int_{w_{0}}^{t} h(w) \Delta w+\int_{w_{0}}^{t} \int_{w_{0}}^{w} K_{1}(s, w, \sigma(s), \sigma(w))\right. \\
& \left.F\left(A^{n-2} u(t)\right) \Delta s \Delta w+\int_{w_{0}}^{t} \int_{w_{0}}^{v_{0}} K_{2}(s, w, \sigma(s), \sigma(w)) G\left(A^{n-2} u(t)\right) \Delta s \Delta w\right) \Delta s \Delta w \\
& +\int_{w_{0}}^{t} \int_{w_{0}}^{v_{0}} K_{2}(s, w, \sigma(s), \sigma(w)) G\left(A^{n-2} u(t)\right) \Delta s \Delta w \\
= & A^{n-1} u(t) . \\
& +\int_{w_{0}}^{t} h(w) \Delta w+\int_{w_{0}}^{t} \int_{w_{0}}^{w} K_{1}(s, w, \sigma(s), \sigma(w)) F\left(A^{n-2} u(t)\right) \Delta s \Delta w \\
& \\
&
\end{aligned}
$$

As a result, we observe that $\left(A^{n} u(t)\right)_{n}$ is strictly decreasing for all $t \in\left[w_{0}, v_{0}\right]$ and a sequence bounded below; hence, it converges to some $L$. As $\left(A_{n}\right)_{n}$ is a monotone sequence, it is known from Dini theorem that $\sup _{t}\left|A^{n} u(t)\right|$ converges to some $L \leq a+\sup _{t}|h(t)|+$ $\sup _{t, s}\left|K_{1}(s, t, \sigma(s), \sigma(t))\right||F(u(s))|+\sup _{t, s}\left|K_{2}(s, t, \sigma(s), \sigma(t))\right||G(u(s))|$. Observe that 


$$
\begin{aligned}
& T\left(u_{n}, u_{m}\right) \frac{R\left(u_{n}, u_{n+1}\right) S\left(u_{n-1}, u_{n}\right)}{T\left(u_{n-1}, u_{n}\right)}=T\left(A^{n} u, A^{m} u\right) \frac{R\left(A^{n} u, A^{n+1} u\right) S\left(A^{n-1} u, A^{n} u\right)}{T\left(A^{n-1} u, A^{n} u\right)} \\
&=\left(1+\sup _{t}\left|A^{n} u(t)-A^{m} u(t)\right|^{2}\right) \times \\
& \frac{\left(66+23 \sup _{t}\left|A^{n-1} u(t)-A^{n} u(t)\right|^{2}\right) \sup _{t}\left\{\left(1+\left|A^{n} u(t)\right|^{2}\right)\left(1+\left|A^{n+1} u(t)\right|^{2}\right)\right\}}{1+\sup _{t}\left|A^{n-1} u(t)-A^{n} u(t)\right|^{2}} \\
& \leq\left(1+\sup _{t}\left\{\left(1+\left|A^{n} u(t)\right|^{2}\right)\left(1+\left|A^{n+1} u(t)\right|^{2}\right)\right\}\right) \times \\
& \frac{\left(66+23 \sup _{t}\left\{\left(1+\left|A^{n} u(t)\right|^{2}\right)\left(1+\left|A^{n+1} u(t)\right|^{2}\right)\right\}\right) \sup _{t}\left\{\left(1+\left|A^{n} u(t)\right|^{2}\right)\left(1+\left|A^{n+1} u(t)\right|^{2}\right)\right\}}{1+\sup _{t}\left|A^{n-1} u(t)-A^{n} u(t)\right|^{2}} \\
& \leq 178\left(1+L^{2}\right)^{6} .
\end{aligned}
$$

Therefore,

$$
\begin{aligned}
\sup _{m} \lim _{n} T\left(u_{n}, u_{m}\right) \frac{R\left(u_{n}, u_{n+1}\right) S\left(u_{n-1}, u_{n}\right)}{T\left(u_{n-1}, u_{n}\right)} \leq & 178\left(1+L^{2}\right)^{6} \\
\leq & 178\left(1+\left[a+\sup _{t}|h(t)|+\sup _{t, s}\left|K_{1}(s, t, \sigma(s), \sigma(t))\right||F(u(s))|\right.\right. \\
& \left.\left.+\sup _{t, s}\left|K_{2}(s, t, \sigma(s), \sigma(t))\right||G(u(s))|\right]^{2}\right)^{6}<\frac{1}{\beta^{2}} .
\end{aligned}
$$

In a similar manner, we can verify the inequalities (5), (6) and (7), respectively. Thereby, $A$ fulfils all the hypotheses of the Theorem 2, the map $A$ described in Equation (19) has a unique fixed point, and the integral Equation (16) has a unique solution in $C\left[w_{0}, v_{0}\right]_{\mathbb{T}}$.

\section{Application}

Theorem 3 is applied to an illustration of Volterra-Fredholm integro-dynamic equations of the second kind in this section.

Example 2. Let $\mathbb{T}=3^{\mathbb{N}_{0}}$. Take into consideration the following nonlinear Volterra-Fredholm integro-dynamic equation:

$$
u^{\Delta}(t)=-\frac{1}{3 t^{2}}-\frac{t^{4}}{4000}-\frac{5597}{12000}+\int_{1}^{t} \frac{\left(1+s^{3}\right)}{100} \frac{1}{1+|u(s)|^{3}} \Delta s+\int_{1}^{9} \frac{\left(2 s^{2}+1\right)}{600} \frac{1}{2+|u(s)|^{2}} \Delta s, \quad t \in[1,9]_{\mathbb{T}},
$$

along with the initial condition $u(1)=3$. Take note of the fact that

$$
\sigma(t)=\inf \left\{s \in \mathbb{T}=3^{\mathbb{N}_{0}}: s>t\right\}=\inf \{3 t, 9 t, \ldots\}=3 t, t \in \mathbb{T} .
$$

In actuality, $u(t)=\frac{1}{t}$ is an immediate solution to the specified nonlinear Volterra integrodynamic Equation (23). Indeed,

$$
u^{\Delta}(t)=\frac{\frac{1}{\sigma(t)}-\frac{1}{t}}{\sigma(t)-t}=\frac{-1}{t \sigma(t)}=\frac{-1}{3 t^{2}}, \quad t \in[1,9]_{\mathbb{T}} .
$$


Let $f(t)=\frac{t^{4}}{10}-\frac{1}{10}$ and $g(t)=\frac{t^{3}}{5}+\frac{1}{5}, t \in[1,9]_{\mathbb{T}}$. The delta derivatives of $f(t)$ and $g(t)$ can then be determined as follows:

$$
f^{\Delta}(t)=\frac{1}{10}\left[\frac{[\sigma(t)]^{4}-t^{4}}{\sigma(t)-t}\right]=\frac{1}{10}(\sigma(t)+t)\left([\sigma(t)]^{2}+t^{2}\right)=4 t^{3},
$$

and

$$
\left.g^{\Delta}(t)=\frac{1}{5}\left[\frac{[\sigma(t)]^{3}-t^{3}}{\sigma(t)-t}\right]=\frac{1}{5}(\sigma(t))^{2}+t \sigma(t)+t^{2}\right)=\frac{13 t^{2}}{5}, \quad t \in[1,9]_{\mathbb{T}} .
$$

Henceforth, the right-hand side of the nonlinear Volterra-Fredholm integro-dynamic Equation (23) is therefore

$$
\begin{aligned}
-\frac{1}{3 t^{2}}-\frac{t^{4}}{4000}-\frac{5597}{12000}+ & \int_{1}^{t} \frac{\left(1+s^{3}\right)}{100} \frac{1}{1+|u(s)|^{3}} \Delta s+\int_{1}^{9} \frac{\left(2 s^{2}+1\right)}{600} \frac{1}{2+|u(s)|^{2}} \Delta s \\
& =-\frac{1}{3 t^{2}}-\frac{t^{4}}{4000}-\frac{5597}{12000}+\int_{1}^{t} \frac{\left(1+s^{3}\right)}{100} \frac{s^{3}}{1+s^{3}} \Delta s+\int_{1}^{9} \frac{\left(2 s^{2}+1\right)}{600} \frac{s^{2}}{2 s^{2}+1} \Delta s \\
& =-\frac{1}{3 t^{2}}-\frac{t^{4}}{4000}-\frac{5597}{12000}+\frac{1}{400} \int_{1}^{t} 4 s^{3} \Delta s+\frac{1}{1560} \int_{1}^{9} \frac{13 s^{2}}{5} \Delta s \\
& =-\frac{1}{3 t^{2}}-\frac{t^{4}}{4000}-\frac{5597}{12000}+\frac{1}{400} \int_{1}^{t} f^{\Delta}(s) \Delta s+\frac{1}{1560} \int_{1}^{9} g^{\Delta}(s) \Delta s \\
& =-\frac{1}{3 t^{2}}-\frac{t^{4}}{4000}-\frac{5597}{12000}+\left.\frac{1}{400} f(s)\right|_{s=1} ^{t}+\left.\frac{1}{1560} g(s)\right|_{s=1} ^{9} \\
& =-\frac{1}{3 t^{2}}-\frac{t^{4}}{4000}-\frac{5597}{12000}+\frac{t^{4}}{4000}-\frac{1}{4000}+\frac{91}{195}=u^{\Delta}(t), \quad t \in[1,9]_{\mathbb{T}} .
\end{aligned}
$$

The map A given in Equation (19) is defined by

$$
\begin{aligned}
A u(t)=3+ & \int_{1}^{t}\left(-\frac{1}{3 t^{2}}-\frac{t^{4}}{4000}-\frac{5597}{12000}\right) \Delta w+\int_{1}^{t} \int_{1}^{w} \frac{\left(1+s^{3}\right)}{100} \frac{1}{1+|u(s)|^{3}} \Delta s \Delta w \\
& +\int_{1}^{t} \int_{1}^{9} \frac{\left(2 s^{2}+1\right)}{600} \frac{1}{2+|u(s)|^{2}} \Delta s \Delta w \quad t \in[1,9]_{\mathbb{T}},
\end{aligned}
$$

so that $K_{1}(s, w, \sigma(s), \sigma(w))=\frac{1+s^{3}}{100}, K_{2}(s, w, \sigma(s), \sigma(w))=\frac{2 s^{2}+1}{600}, F(u(s))=\frac{1}{1+|u(s)|^{3}}$ and $G(u(s))=\frac{1}{2+|u(s)|^{2}}$. The Cauchy-Schwarz inequality and the definition of the map $A$ in Equation (24) lead to the conclusion that

$$
\begin{aligned}
|A u(t)-A z(t)|^{2}= & (A u(t)-A z(t))^{2} \\
= & {\left[\int_{1}^{t} \int_{1}^{w} \frac{\left(1+s^{3}\right)}{100}\left[\frac{1}{1+|u(s)|^{3}}-\frac{1}{1+|z(s)|^{3}}\right] \Delta s \Delta w+\int_{1}^{t} \int_{1}^{9} \frac{\left(2 s^{2}+1\right)}{600}\left[\frac{1}{2+|u(s)|^{2}}-\frac{1}{2+|z(s)|^{2}}\right] \Delta s \Delta w\right]^{2} } \\
\leq & 2\left(\int_{1}^{t} \int_{1}^{w} \frac{\left(1+s^{3}\right)^{2}}{(100)^{2}} \Delta s \Delta w\right)\left(\int_{1}^{t} \int_{1}^{w}\left|\frac{1}{1+|u(s)|^{3}}-\frac{1}{1+|z(s)|^{3}}\right|^{2} \Delta s \Delta w\right)+ \\
& 2\left(\int_{1}^{t} \int_{1}^{t} \frac{\left(2 s^{2}+1\right)^{2}}{(600)^{2}} \Delta s \Delta w\right)\left(\int_{1}^{t} \int_{1}^{9}\left|\frac{1}{2+|u(s)|^{2}}-\frac{1}{2+|z(s)|^{2}}\right|^{2} \Delta s \Delta w\right) .
\end{aligned}
$$




\section{Observe that}

$$
\begin{aligned}
\left|\frac{1}{1+|u(s)|^{3}}-\frac{1}{1+|z(s)|^{3}}\right|^{2} & =\left|\frac{|u(s)|^{3}-|z(s)|^{3}}{\left(1+|u(s)|^{3}\right)\left(1+|z(s)|^{3}\right)}\right|^{2} \\
& \leq\left.|| u(s)|-| z(s)\right|^{2}\left[\frac{|u(s)|^{2}+2|u(s)||z(s)|+|z(s)|^{2}}{1+|u(s)|^{3}+|z(s)|^{3}+|u(s)|^{3}|z(s)|^{3}}\right]^{2} \\
& \leq\left.|| u(s)|-| z(s)\right|^{2}\left[\frac{(|u(s)|+|z(s)|)^{2}}{1+|u(s)|^{3}+|z(s)|^{3}}\right]^{2} \\
& =|| u(s)|-| z(s)||^{2}\left[\frac{4|u(s)||z(s)|+(|u(s)|-|z(s)|)^{2}}{1+|u(s)|^{3}+|z(s)|^{3}}\right]^{2} \\
& =|| u(s)-\left.z(s)\right|^{2} \frac{\left[16|u(s)|^{2}|z(s)|^{2}+|| u(s)|-| z(s)||^{4}+\left.8|| u(s)|-| z(s)\right|^{2}|u(s)||z(s)|\right]}{\left[1+|u(s)|^{3}+|z(s)|^{3}\right]^{2}},
\end{aligned}
$$

$$
\begin{aligned}
\left|\frac{1}{2+|u(s)|^{2}}-\frac{1}{2+|z(s)|^{2}}\right|^{2} & =|| u(s)|-| z(s)||^{2}\left[\frac{|u(s)|+|z(s)|}{\left(2+|u(s)|^{2}\right)\left(2+|z(s)|^{2}\right)}\right]^{2} \\
& =\left.|| u(s)|-| z(s)\right|^{2} \frac{\left[4|u(s)||z(s)|+(|u(s)|-|z(s)|)^{2}\right]}{\left[4+2|u(s)|^{2}+2|z(s)|^{2}+|u(s)|^{2}|z(s)|^{2}\right]^{2}} \\
& \leq|| u(s)|-| z(s)||^{2} \frac{\left[4|u(s)||z(s)|+|| u(s)|-| z(s)||^{2}\right]}{\left[4+2|u(s)|^{2}+2|z(s)|^{2}\right]^{2}} .
\end{aligned}
$$

Consequently, Equation (25) becomes

$$
\begin{aligned}
|A u(t)-A z(t)|^{2} \leq & \left(\int_{1}^{t} \int_{1}^{w} \frac{\left(1+s^{3}\right)^{2}}{5000} \Delta s \Delta w\right)\left(\int_{1}^{t} \int_{1}^{w}|u(s)-z(s)|^{2}\right. \\
& \left.\times \frac{\left[16|u(s)|^{2}|z(s)|^{2}+|u(s)-z(s)|^{4}+8|u(s)-z(s)|^{2}|u(s)||z(s)|\right]}{\left[1+|u(s)|^{3}+|z(s)|^{3}\right]^{2}} \Delta s \Delta w\right) \\
& +\left(\int_{1}^{t} \int_{1}^{9} \frac{\left(2 s^{2}+1\right)^{2}}{180000} \Delta s \Delta w\right)\left(\int_{1}^{t} \int_{1}^{9}|u(s)-z(s)|^{2} \frac{\left[4|u(s)||z(s)|+|u(s)-z(s)|^{2}\right]}{\left[4+2|u(s)|^{2}+2|z(s)|^{2}\right]^{2}} \Delta s \Delta w\right) .
\end{aligned}
$$

Thereafter, by evaluating the delta integral and utilizing the fact that $|t| \leq 9$, we acquire 


$$
\begin{aligned}
& |A u(t)-A z(t)|^{2} \leq \frac{1}{5000}\left(\frac{t^{8}}{3585040}+\frac{t^{5}}{2420}+\frac{t^{2}}{4}-\frac{22973 t}{21860}-\frac{108606989}{433789840}\right)\left(\int_{1}^{t} \int_{1}^{w}|u(s)-z(s)|^{2}\right. \\
& \left.\times\left[\frac{16|u(s)|^{2}|z(s)|^{2}+|u(s)-z(s)|^{4}+8|u(s)-z(s)|^{2}|u(s)||z(s)|}{\left[1+|u(s)|^{3}+|z(s)|^{3}\right]^{2}}\right] \Delta s \Delta w\right) \\
& +\frac{17472}{180000}\left(\int_{1}^{t} \int_{1}^{9}|u(s)-z(s)|^{2}\left[\frac{4|u(s)||z(s)|+|u(s)-z(s)|^{2}}{\left[4+2|u(s)|^{2}+2|z(s)|^{2}\right]^{2}}\right] \Delta s \Delta w\right) \\
& \leq \frac{46.949}{5000} \int_{1}^{t} \int_{1}^{w}|u(s)-z(s)|^{2}\left[\frac{16|u(s)|^{2}|z(s)|^{2}+|u(s)-z(s)|^{4}+8|u(s)-z(s)|^{2}|u(s)||z(s)|}{\left[1+|u(s)|^{3}+|z(s)|^{3}\right]^{2}}\right] \Delta s \Delta w \\
& +\frac{17472}{180000}\left(\int_{1}^{t} \int_{1}^{9}|u(s)-z(s)|^{2}\left[\frac{4|u(s)||z(s)|+|u(s)-z(s)|^{2}}{\left[4+2|u(s)|^{2}+2|z(s)|^{2}\right]^{2}}\right] \Delta s \Delta w\right. \\
& \leq F_{1} \int_{1}^{t} \int_{1}^{w}|u(s)-z(s)|^{2} \\
& {\left[\frac{16\left(1+|u(s)|^{2}\right)\left(1+|z(s)|^{2}\right)+|u(s)-z(s)|^{2}\left(1+|u(s)|^{2}\right)\left(1+|z(s)|^{2}\right)+8|u(s)-z(s)|^{2}\left(1+|u(s)|^{2}\right)\left(1+|z(s)|^{2}\right)}{1+|u(s)-z(s)|^{2}}\right] \Delta s \Delta w} \\
& +2 F_{2}\left(\int_{1}^{t} \int_{1}^{9}|u(s)-z(s)|^{2}\left[\frac{4\left(1+|u(s)|^{2}\right)\left(1+|z(s)|^{2}\right)+|u(s)-z(s)|^{2}}{1+|u(s)-z(s)|^{2}}\right] \Delta s \Delta w\right.
\end{aligned}
$$

where $F_{1}=\frac{46.949}{5000}$ and $F_{2}=\frac{8736}{180000}$. When the supremum over $t \in[1,9]_{\mathbb{T}}$ is combined with the metric's definition given in Equation (17), one obtains

$$
\begin{gathered}
d_{\mathrm{T}}(A u, A z) \leq \frac{\sup _{t \in[1,9]_{\mathbb{T}}}\left\{\left(1+|u(t)|^{2}\right)\left(1+|z(t)|^{2}\right)\right\}}{1+\sup _{t \in[1,9]_{\mathbb{T}}}|u(t)-z(t)|^{2}}\left[F_{1}\left(16+9 \sup _{t \in[1,9]_{\mathbb{T}}}|u(t)-z(t)|^{2}\right) \int_{1}^{t} \int_{1}^{w} \Delta s \Delta w\right. \\
\left.+2 F_{2}\left(4+\sup _{t \in[1,9]_{\mathbb{T}}}|u(t)-z(t)|^{2}\right) \int_{1}^{t} \int_{1}^{9} \Delta s \Delta w\right] d_{\mathrm{T}}(u, z) .
\end{gathered}
$$

By Theorem 3, we have

$$
\begin{aligned}
d_{\mathrm{T}}(A u, A z) \leq & \frac{\sup _{t \in[1,9]_{\mathbb{T}}}\left(\left(1+|u(t)|^{2}\right)\left(1+|z(t)|^{2}\right)\right)}{1+\sup _{t \in[1,9]_{\mathbb{T}}}|u(t)-z(t)|^{2}}\left[F_{1}\left(16+9 \sup _{t \in[1,9]_{\mathbb{T}}}|u(t)-z(t)|^{2}\right) \frac{(t-1)^{2}}{2}\right. \\
& \left.+128 F_{2}\left(4+\sup _{t \in[1,9]_{\mathbb{T}}}|u(t)-z(t)|^{2}\right)\right] d_{\mathrm{T}}(u, z),
\end{aligned}
$$


where $F_{1}=\frac{46.949}{5000}$ and $t, w \in[1,9]_{\mathbb{T}}$, i.e., $|t| \leq 9$ and $|w| \leq 9$, then $F_{1} \frac{(t-1)^{2}}{2} \leq \frac{46.949}{5000} \cdot \frac{64}{2}<\frac{1}{2}$ and $128 F_{2}=128 \cdot \frac{8736}{180000}=6.21$. Hence, the equation is further transformed into

$$
\begin{aligned}
d_{\mathrm{T}}(A u, A z) & \leq \frac{1}{2} \frac{\left(66+23 \sup _{t \in[1,9]_{\mathbb{T}}}|u(t)-z(t)|^{2}\right) \sup _{t \in[1,9]_{\mathbb{T}}}\left(\left(1+|u(t)|^{2}\right)\left(1+|z(t)|^{2}\right)\right)}{1+\sup _{t \in[1,9]_{\mathbb{T}}}|u(t)-z(t)|^{2}} d_{\mathrm{T}}(u, z) \\
& =\beta \frac{R(u, z) S(u, z)}{T(u, z)} d_{\mathrm{T}}(u, z)
\end{aligned}
$$

where $\beta=\frac{1}{2}, R(u, z)=66+23 \sup _{t \in[1,9]_{\mathbb{T}}}|u(t)-z(t)|^{2}, S(u, z)=\sup _{t \in[1,9]_{\mathbb{T}}}\left(\left(1+|u(t)|^{2}\right)(1+\right.$ $\left.\left.|z(t)|^{2}\right)\right)$ and $T(u, z)=1+\sup _{t \in[1,9]_{\mathbb{T}}}|u(t)-z(t)|^{2}$. Thereby, the map A specified in Equation (24) has a unique fixed point by Theorem 3, i.e., the integral equation stated in Equation (23) has a unique solution in $C[1,9]_{\mathbb{T}}$.

\section{Conclusions}

This article explores the existence and uniqueness of solutions for a class of nonlinear Volterra-Fredholm integro-dynamic equations of the second kind on time scales. We approach the problem in the context of triple controlled metric type spaces, which adopts a different perspective on its solution. Eventually, we demonstrate an example to ensure the existence of a unique solution to an integro-dynamic problem. In future studies, we can extend this technique to higher-order delta derivatives with fixed point theorems of different types to acquire further general conditions for the existence and uniqueness of IVPs correlated with Volterra-Fredholm integro-dynamic equations on time scales.

Author Contributions: Conceptualization, K.G., S.T.Z. and T.A.; methodology, S.T.Z., T.A. and N.M.; validation, T.A. and N.M.; investigation, T.A. and N.M.; writing-original draft preparation, K.G. and S.T.Z; writing-review and editing, K.G. and S.T.Z.; supervision, K.G.; funding acquisition, T.A. and N.M. All authors have read and agreed to the published version of the manuscript.

Funding: This research received no external funding.

Data Availability Statement: Not applicable.

Acknowledgments: The authors Kalpana Gopalan and Sumaiya Tasneem Zubair would like to thank the Management of Sri Sivasubramaniya Nadar College of Engineering for their continuous support and encouragement to carry out this research work. The authors Thabet Abdeljawad and Nabil Mlaiki would like to thank Prince Sultan University for paying the APC and for the support through the TAS research lab.

Conflicts of Interest: The authors declare no conflict of interest.

\section{References}

1. Hilger, S. Ein Maßkettenkalkül mit Anvendung auf Zentrumsmannigfaltigkeiten. Ph.D. Thesis, Universität at Würzburg, Würzburg, Germany, 1988.

2. Agarwal, R.P.; Bohner, M.; Peterson, A. Inequalities on time scales: A survey. Math. Inequalities Appl. 2001, 4, 535-557. [CrossRef]

3. Guseinov, G.S. Integration on time scales. J. Math. Anal. Appl. 2003, 285, 107-127. [CrossRef]

4. Georgiev, S. Integral Equations on Time Scales; Atlantis Press: Paris, France, 2016.

5. Georgiev, S. Fractional Dynamic Calculus and Fractional Dynamic Equations on Time Scales; Springer: Cham, Switzerland, 2018.

6. Hilger, S. Analysis on measure chains-a unified approach to continuous and discrete calculus. Results Math. 1990, $18,18-56$. [CrossRef]

7. Bohner, M.; Peterson, A. Dynamic Equations on Time Scales: An Introduction with Applications; Birkhäuser: Boston, MA, USA, 2003.

8. Atalan, Y.; Karakaya, V. Stability of nonlinear Volterra-Fredholm integro differential equation: A fixed point approach. Creat. Math. Inform. 2017, 26, 247-254. [CrossRef]

9. Marzban, H.R.; Hoseini, S.M. Solution of nonlinear Volterra-Fredholm integro differential equations via hybrid of block-pulse functions and lagrange interpolating polynomials. Adv. Numer. Anal. 2012, 2012, 868279. [CrossRef] 
10. Araghi, M.A.F.; Behzadi, S.H.S. Solving nonlinear Volterra-Fredholm integro-differential equations using the modified adomian decomposition method. Comput. Methods Appl. Math. 2009, 9, 321-331. [CrossRef]

11. Al-Saar, F.M.; Ghadle, K.P. Solving nonlinear Fredholm integro-differential equations via modifications of some numerical methods. Adv. Theory Nonlinear Anal. Its Appl. 2021, 5, 260-276. [CrossRef]

12. Hamoud, A.; Mohammed, N.; Ghadle, K. Existence and uniqueness results for Volterra-Fredholm integro-differential equations. Adv. Theory Nonlinear Anal. Its Appl. 2020, 4, 361-372. [CrossRef]

13. Harikrishnan, S.; Kanagarajan, K.; Vivek, D. Some existence and stability results for integro-differential equation by hilferkatugampola fractional derivative. Palest. J. Math. 2020, 9, 254-262.

14. Malar, K.; Anguraj, A. Existence results for fractional integrodifferential systems with interval impulse via sectorial operator. Results Nonlinear Anal. 2019, 2, 169-181.

15. Sudsutad, W.; Alzabut, J.; Nontasawatsri, S.; Thaiprayoon, C. Stability analysis for a generalized proportional fractional Langevin equation with variable coefficient and mixed integro-differential boundary conditions. J. Nonlinear Funct. Anal. 2020, $2020,23$.

16. Houas, M.; Bezziou, M. Existence of solutions for neutral Caputo-type fractional integro-differential equations with nonlocal boundary conditions. Commun. Optim. Theory 2021, 2021, 9.

17. Svinik-Adıgüzel, R.; Karapınar, E.; Erhan, I.M. A Solution to nonlinear Volterra integro-dynamic equations via fixed point theory. Filomat 2019, 33, 5333-5345. [CrossRef]

18. Kamran, T.; Samreen, M.; Ain, Q.U.L. A generalization of $b$-metric space and some fixed point theorems. Mathematics 2017, 5, 19. [CrossRef]

19. Mlaiki, N.; Aydi, H.; Souayah, N.; Abdeljawad, T. Controlled metric type spaces and the related contraction principle. Mathematics 2018, 6, 194. [CrossRef]

20. Abdeljawad, T.; Mlaiki, N.; Aydi, H.; Souayah, N. Double controlled metric type spaces and some fixed point results. Mathematics 2018, 6, 320. [CrossRef]

21. Tasneem, Z.S.; Kalpana, G.; Abdeljawad, T. Controlled b-Branciari metric type spaces and related fixed point theorems with applications. Filomat 2020, 34, 4253-4269.

22. Tasneem, Z.S.; Kalpana, G.; Abdeljawad, T. A different approach to fixed point theorems on triple controlled metric type spaces with a numerical experiment. Dyn. Syst. Appl. 2021, 30, 111-130.

23. Kalpana, G.; Tasneem, Z.S. Some fixed point results in extended hexagonal $b$-metric spaces approach to the existence of a solution to fredholm integral equations. J. Math. Anal. 2020, 11, 1-17.

24. Abdeljawad, T.; Abdalla, B. Monotonicity results for delta and nabla Caputo and Reimann fractional differences via dual identities. Filomat 2017, 31, 3671-3683. [CrossRef]

25. Frechet, M. Sur quelques points duo calcul fonctionel. Rendicouti Mah. Palermo 1906, 22, 1-72. [CrossRef]

26. Ali, M.U.; Kamran, T.; Postolache, M. Solution of Volterra integral inclusion in $b$-metric spaces via new fixed point theorem. Nonlinear Anal. Model. Control. 2017, 22, 17-30. [CrossRef]

27. Abodayeh, K.; Karapınar, E.; Pitea, A.; Shatanawi, W. Hybrid contractions on Branciari type distance spaces. Mathematics 2019, 7 , 994. [CrossRef]

28. Kamran, T.; Postolache, M.; Ali, M.U.; Kiran, Q. Feng and Liu type F-contraction in $b$-metric spaces with an appliation to integral equations. J. Math. Anal. 2016, 7, 18-27.

29. Kalpana, G.; Tasneem, Z.S. Existence of fixed point results in $C^{*}$-algebra-valued triple controlled metric type spaces. In Proceedings of the Mathematical Analysis and Computing: ICMAC 2019, Kalavakkam, India, 23-24 December 2019. [CrossRef]

30. Salman, F.; Hüseyin, I.; Saleem, N. Fuzzy triple controlled metric spaces and related fixed point results. J. Funct. Spaces 2021, 2021, 9936992.

31. Mlaiki, N.; Abdeljawad, T.; Shatanawi, W.; Aydi, H.; Gaba, Y.U. On complex-valued triple controlled metric spaces and applications. J. Funct. Spaces 2021, 2021, 5563456. [CrossRef] 